\title{
Those with the habit of going to sleep early show a higher ratio of lympho- cytes while those with the habit of staying up late show a higher ratio of granulocytes
}

\author{
Kiyoshi ADACHI, ${ }^{1,2}$, Kazushi NishiJO ${ }^{2}$ and Toru ABo ${ }^{1}$ \\ ${ }^{1}$ Department of Immunology, Niigata University School of Medicine, Niigata 951-8510, Japan and ${ }^{2}$ The Graduate University of Japan \\ Traditional Medicine and Science, Tokyo 160-0017, Japan \\ (Received 18 January 2010; and accepted 15 February 2010)
}

\begin{abstract}
In this study, we examined the effect of the difference in time of going to sleep on the numerical values of leukocyte subsets and various hormones. Subjects consisted of 26 healthy adults (15 men, 11 women) with a mean age of 37.6 years. Among the 26 individuals, 12 persons (Group E) were of the habit of going to sleep before midnight consistently, while 14 persons (Group L) were of the habit of staying up late, consistently going to sleep after 2 am. For Group E, it was found that the ratio of lymphocytes was remarkably high in comparison with Group L (Group E 41.6 $2.54 \%$, Group L $31.7 \pm 2.03 \%, P<0.01$ ). On the other hand, for Group L it was found that the ratio of granulocytes was remarkably high in comparison with Group E (Group E $53.0 \pm 2.51 \%$, Group L $62.3 \pm 2.22 \%, P<0.01$ ). However, no difference was observed in lymphocyte and granulocyte ratios due to the duration of the sleep. As the excessive quantity of granulocytes was not corrected through longer sleep, these findings suggest that the time when first going to sleep is more important than the total hours of sleep achieved.
\end{abstract}

Human beings lived for many generations according the rhythms of the natural world, going to sleep when the sun set in the evening and waking up when the sun rose in the morning. However, with the spread of artificial electrical illumination, this natural rhythm has changed. Research into the effects of these changes, the introduction of disorder into the previously orderly, natural practices of sleeping and awaking, on the autonomic nervous system is of quite some interest. Furthermore, some research has indicated that the autonomic nervous system may in fact control the amount of leukocytes present in the blood (3). Receptors for adrenaline do exist on the membranes of leukocytes (2), and it has

Address correspondence to: Kiyoshi Adachi, D.D.S., The Graduate University of Japan Traditional Medicine and Science, 5 Samon-cho, Shinjuku-ku, Tokyo, 160-0017, Japan

Tel: +81-3-3352-6811, Fax: +81-3-3352-6816

E-mail: adachi@dento-iryo.ac.jp been found in mice that the amount of granulocytes does increase after injections of adrenaline (4). In this research, we investigated the possible influence of the difference in time of going to sleep upon quantities of leukocyte subsets and various hormones. Up to this time, reports of such investigation on the relationship between time of going to sleep, or length of time spent sleeping, and amount of leukocyte subsets and various hormones have been few in number.

\section{MATERIALS AND METHODS}

Subjects and ethics. This study was carried out with the approval of Ethical Committee of The Graduate University of Japan Traditional Medicine and Science. The 26 subjects signed forms of consent after receiving sufficient explanation of the objectives and possible risks of participation. The 26 subjects were all healthy Japanese persons. The average age of the 26 subjects was $37.6 \pm 2.58$ (SE) years, and the 12 
individuals with the consistent habit of going to sleep before midnight were grouped into Group E, while the 14 individuals with the consistent habit of staying up late past 2 am were grouped into Group L. The setting of the early sleeping time at midnight was made in consideration of the habit of the subjects, residents of the Tokyo metropolitan area. There were 15 men (Group E: 7, Group L: 8) and 11 women (Group E: 5, Group L: 6).

Blood samples and analysis. Quantities of leukocyte subsets and various hormones, as well as blood glucose concentration, were all measured from blood taken from the forearm median antebrachial vein in all subjects, and the blood was taken while subjects were resting in the seated position. Body temperature was measured under the arm (axillary) at the time of drawing blood. In consideration of daily rhythms, blood samples were taken between $4: 30$ pm and 5:30 pm. Also, in all cases blood samples were taken at least $4 \mathrm{~h}$ after eating. From each subject $25 \mathrm{~mL}$ of blood was taken, and then immediately processed by centrifuge. After centrifuging, the blood plasma was immediately frozen and then analyzed by Mitsubishi Chemical Medience Corporation, Tokyo, Japan. The methods of analysis are as follows:

Leukocyte subsets (stab nuclear leukocyte, segmented nuclear leukocyte, eosinophil, basophil, monocyte, lymphocyte): microscopical test

Glucose: enzymatic assay

$\mathrm{GH}$ : immunoradiometric assay (beads solid phase method)

ACTH: immunoradiometric assay (beads solid phase method)

TSH: chemiluminescent immunoassay

FT3 (free triiodothyronine): chemiluminescent immunoassay

Cortisol: radioimmunoassay (coated tube solid phase method)

Noradrenaline: high performance liquid chromatography

Among leukocyte subsets, stab nuclear leukocyte, segmented nuclear leukocyte, eosinophil, and basophil are together called granulocytes. The numbers of lymphocytes and granulocytes were calculated by multiplying the ratios of each respectively with the total number of white blood cells.

Statistical analysis. Figures are all shown as MEAN \pm SE. Statistical analysis was performed by twotailed Student's $t$ test. In the case that $P$ was less than 0.05 the difference was taken to be statistically significant and indicated by $*$. In the case that $P$ was less than 0.01 the difference was taken to be particularly statistically significant and indicated by $* *$.

\section{RESULTS}

The leukocyte subsets, blood glucose levels, hormone levels, under the arm (axillary) body temperatures, average times of going to sleep, and average length of time slept for the 26 subjects are shown in Table 1. Table 2 shows the results of comparing and analyzing Group E (E-01 to E-12) with Group L (L-01 to L-14) from Table 1. Furthermore, measures that were found statistically significant, as well as a graph of numbers of leukocytes are shown in Fig. 1.

Differences in leukocyte subsets among individuals with habit of going to sleep early and those with habit of staying up late

Regarding leukocyte number (WBC), for Group E the finding was $6.0 \pm 0.31\left(\times 10^{3} / \mu \mathrm{L}\right)$, and for Group $\mathrm{L}$ the finding was $6.7 \pm 0.41\left(\times 10^{3} / \mu \mathrm{L}\right)$. This did not represent a statistically significant difference. However, for ratio of lymphocytes, Group E was $41.6 \pm$ $2.54 \%$, and Group L was found to be $31.7 \pm 2.03 \%$, with Group E clearly showing a higher ratio $(P<$ $0.01)$. For ratio of granulocytes, Group $\mathrm{E}$ showed $53.0 \pm 2.51 \%$, while Group L was $62.3 \pm 2.22 \%$. Here, Group L showed a clearly higher ratio $(P<$ $0.01)$. Then, multiplying this ratio by number of leukocytes and comparing number of lymphocytes with number of granulocytes, for number of lymphocytes Group E was $2.5 \pm 0.17\left(\times 10^{3} / \mu \mathrm{L}\right)$, while Group L was $2.1 \pm 0.13\left(\times 10^{3} / \mu \mathrm{L}\right)$, with Group E showing statistically significantly greater number. For number of granulocytes, Group E was $3.2 \pm 0.27\left(\times 10^{3} / \mu \mathrm{L}\right)$ and Group L was $4.2 \pm 0.36\left(\times 10^{3} / \mu \mathrm{L}\right)$, with Group L showing a statistically significantly greater number. However, all amounts were within the standard range. Thus, we found that for those with the habit of regularly going to sleep early, among leukocyte subsets, the ratio and number of lymphocytes was greater, while for those with the habit of staying up late, the ratio and number of granulocytes was greater (Table 2).

Differences in blood glucose and hormone levels among individuals with habit of going to sleep early and those with habit of staying up late

For blood glucose level, Group E was found to be $95 \pm 2.4 \mathrm{mg} / \mathrm{dL}$, and Group L was $88 \pm 2.7 \mathrm{mg} / \mathrm{dL}$, with Group E being significantly higher (Table 2). However, for the hormones tested and also for body 
Table 1 Individual values of hematologic parameters, body temperatures, average times of going to sleep and average length of time slept in individuals with habit of going to sleep before midnight and those with habit of staying up late

\begin{tabular}{|c|c|c|c|c|c|c|c|c|c|c|c|c|c|c|c|c|c|c|c|c|c|c|}
\hline \multirow[b]{2}{*}{ Group } & \multirow[b]{2}{*}{ Age } & \multirow[b]{2}{*}{ Sex } & \multirow[b]{2}{*}{$\begin{array}{l}\text { WBC } \\
10^{3} / \mu \mathrm{L}\end{array}$} & \multirow[b]{2}{*}{ Stab. } & \multicolumn{5}{|c|}{$\%$ leukocyte subset count (\%) } & \multicolumn{3}{|c|}{ Number } & \multirow[b]{2}{*}{$\begin{array}{c}\text { Glucose } \\
\mathrm{mg} / \mathrm{dL}\end{array}$} & \multicolumn{6}{|c|}{ Hormone } & \multirow[b]{2}{*}{$\begin{array}{l}\text { Body } \\
\text { Temp. } \\
{ }^{\circ} \mathrm{C}\end{array}$} & \multirow[b]{2}{*}{$\begin{array}{c}\text { Time of } \\
\text { going to } \\
\text { sleep }\end{array}$} & \multirow[b]{2}{*}{$\begin{array}{l}\text { Length } \\
\text { of time } \\
\text { slept(h) }\end{array}$} \\
\hline & & & & & Seg. & Eos. & Baso. & Mono & Lym. & Gran. & $\begin{array}{c}\text { Lym. } \\
10^{3} / \mu \mathrm{L}\end{array}$ & $\begin{array}{c}\text { Gran. } \\
10^{3} / \mu \mathrm{L}\end{array}$ & & $\underset{\mathrm{ng} / \mathrm{mL}}{\mathrm{GH}}$ & $\begin{array}{l}\mathrm{ACTH} \\
\mathrm{pg} / \mathrm{mL}\end{array}$ & $\begin{array}{c}\text { TSH } \\
\mu \mathrm{IU} / \mathrm{mL}\end{array}$ & $\begin{array}{c}\text { FT3 } \\
\mathrm{pg} / \mathrm{mL}\end{array}$ & $\begin{array}{c}\text { Cortisol } \\
\mu \mathrm{g} / \mathrm{dL}\end{array}$ & $\begin{array}{c}\mathrm{NA} \\
\mathrm{ng} / \mathrm{mL}\end{array}$ & & & \\
\hline E-01 & 57 & $\mathrm{~F}$ & 5.6 & 2.5 & 37.5 & 1.5 & 0.5 & 4.5 & 53.5 & 42.0 & 3.0 & 2.4 & 94 & 0.06 & 16 & 3.01 & 3.2 & 6.7 & 0.83 & 36.3 & $24: 00$ & 6.0 \\
\hline E-02 & 21 & M & 6.7 & 4.0 & 36.0 & 6.0 & 0.0 & 8.0 & 46.0 & 46.0 & 3.1 & 3.1 & 104 & 0.14 & 47 & 1.57 & 3.1 & 18.6 & 0.29 & 36.7 & $24: 00$ & 6.0 \\
\hline E-03 & 40 & M & 5.3 & 1.0 & 45.5 & 1.0 & 0.0 & 8.0 & 44.5 & 47.5 & 2.4 & 2.5 & 91 & 0.34 & 52 & 1.86 & 3.5 & 13.0 & 0.36 & 35.7 & $24: 00$ & 7.0 \\
\hline E-04 & 34 & M & 7.6 & 2.0 & 57.0 & 4.5 & 0.0 & 4.5 & 32.0 & 63.5 & 2.4 & 4.8 & 96 & 0.03 & 17 & 0.68 & 3.5 & 5.9 & 0.28 & 36.7 & $22: 30$ & 5.5 \\
\hline E-05 & 30 & $\mathrm{~F}$ & 4.9 & 3.0 & 56.0 & 0.5 & 0.5 & 6.0 & 34.0 & 60.0 & 1.7 & 2.9 & 84 & 0.41 & 13 & 1.49 & 2.7 & 8.2 & 0.33 & 36.9 & $24: 00$ & 6.5 \\
\hline E-06 & 53 & F & 4.4 & 1.0 & 43.5 & 1.5 & 1.0 & 5.5 & 47.5 & 47.0 & 2.1 & 2.1 & 97 & 0.09 & 18 & 1.08 & 3.1 & 9.9 & 0.42 & 36.7 & $24: 00$ & 6.0 \\
\hline E-07 & 45 & $\mathrm{~F}$ & 6.8 & 1.0 & 59.0 & 5.0 & 1.0 & 4.0 & 30.0 & 66.0 & 2.0 & 4.5 & 97 & 0.88 & 25 & 1.83 & 3.0 & 10.4 & 0.28 & 36.7 & $24: 00$ & 7.5 \\
\hline E-08 & 45 & $\mathrm{~F}$ & 5.4 & 1.0 & 49.0 & 2.0 & 0.0 & 5.0 & 43.0 & 52.0 & 2.3 & 2.8 & 106 & 0.08 & 42 & 2.12 & 3.2 & 20.4 & 0.26 & 37.5 & $24: 00$ & 5.0 \\
\hline E-09 & 59 & M & 7.6 & 1.0 & 53.0 & 2.0 & 0.0 & 3.0 & 41.0 & 56.0 & 3.1 & 4.3 & 97 & 0.05 & 77 & 1.62 & 3.1 & 11.7 & 0.41 & 36.0 & $23: 30$ & 6.0 \\
\hline E-10 & 56 & M & 5.4 & 0.5 & 40.0 & 10.5 & 1.5 & 4.0 & 43.5 & 52.5 & 2.3 & 2.8 & 84 & 1.21 & 17 & 3.03 & 2.6 & 4.2 & 0.37 & 36.0 & $23: 00$ & 8.0 \\
\hline E-11 & 49 & M & 6.3 & 1.0 & 38.0 & 3.0 & 0.0 & 4.0 & 54.0 & 42.0 & 3.4 & 2.6 & 106 & 0.09 & 21 & 1.04 & 3.4 & 14.6 & 0.35 & 36.4 & $24: 00$ & 8.0 \\
\hline E-12 & 38 & $\mathrm{M}$ & 6.3 & 1.0 & 56.0 & 3.0 & 1.0 & 9.0 & 30.0 & 61.0 & 1.9 & 3.8 & 86 & 0.26 & 13 & 2.11 & 3.3 & 9.8 & 0.39 & 36.3 & $23: 00$ & 6.0 \\
\hline L-01 & 51 & $\mathrm{M}$ & 7.9 & 4.5 & 62.5 & 1.5 & 0.0 & 5.0 & 26.5 & 68.5 & 2.1 & 5.4 & 79 & 0.04 & 24 & 1.46 & 3.3 & 8.5 & 0.43 & 36.2 & $3: 00$ & 6.0 \\
\hline L-02 & 19 & M & 7.0 & 3.5 & 51.5 & 3.5 & 0.0 & 4.5 & 37.0 & 58.5 & 2.6 & 4.1 & 92 & 0.15 & 15 & 4.36 & 3.5 & 8.3 & 0.36 & 36.8 & $5: 00$ & 4.5 \\
\hline L-03 & 31 & M & 10.0 & 3.0 & 54.5 & 6.5 & 0.0 & 5.5 & 30.5 & 64.0 & 3.1 & 6.4 & 84 & 0.04 & 23 & 0.62 & 3.4 & 6.4 & 0.36 & 37.1 & $2: 30$ & 7.5 \\
\hline L-04 & 54 & M & 5.8 & 2.5 & 56.0 & 1.5 & 0.0 & 4.0 & 36.0 & 60.0 & 2.1 & 3.5 & 90 & 0.18 & 29 & 1.31 & 3.1 & 10.5 & 0.26 & 37.2 & $2: 00$ & 5.0 \\
\hline L-05 & 51 & $\mathrm{~F}$ & 7.7 & 3.5 & 69.0 & 2.5 & 0.0 & 5.0 & 20.0 & 75.0 & 1.5 & 5.8 & 88 & 2.04 & 12 & 0.80 & 2.7 & 8.2 & 0.51 & 35.7 & $2: 00$ & 7.0 \\
\hline L-06 & 20 & M & 6.3 & 1.0 & 50.0 & 2.0 & 0.0 & 6.5 & 40.5 & 53.0 & 2.6 & 3.3 & 100 & 0.18 & 19 & 1.13 & 3.5 & 9.6 & 0.28 & 36.9 & $3: 00$ & 7.0 \\
\hline L-07 & 29 & M & 6.8 & 4.0 & 65.5 & 3.5 & 0.5 & 6.0 & 20.0 & 73.5 & 1.4 & 5.0 & 108 & 0.04 & 21 & 0.97 & 3.7 & 11.9 & 0.31 & 36.7 & $3: 00$ & 5.0 \\
\hline L-08 & 24 & M & 5.9 & 1.5 & 46.5 & 6.0 & 1.0 & 9.5 & 35.5 & 55.0 & 2.1 & 3.2 & 77 & 0.36 & 41 & 1.65 & 3.1 & 15.7 & 0.73 & 36.8 & $4: 00$ & 7.0 \\
\hline L-09 & 25 & $\mathrm{~F}$ & 6.1 & 4.0 & 51.0 & 4.0 & 0.0 & 5.0 & 36.0 & 59.0 & 2.2 & 3.6 & 83 & 0.16 & 24 & 1.66 & 3.2 & 10.8 & 0.17 & 35.4 & $5: 30$ & 5.5 \\
\hline L-10 & 26 & M & 5.5 & 2.5 & 65.0 & 0.0 & 0.5 & 5.5 & 26.5 & 68.0 & 1.5 & 3.7 & 68 & 0.24 & 15 & 1.54 & 3.5 & 13.2 & 0.39 & 37.0 & $2: 00$ & 7.0 \\
\hline L-11 & 31 & $\mathrm{~F}$ & 5.9 & 1.0 & 54.0 & 1.0 & 0.0 & 9.0 & 35.0 & 56.0 & 2.1 & 3.3 & 94 & 0.15 & 18 & 2.86 & 2.8 & 14.4 & 0.27 & 36.4 & $3: 00$ & 7.0 \\
\hline L-12 & 28 & $\mathrm{~F}$ & 4.0 & 3.0 & 52.5 & 1.0 & 1.0 & 4.5 & 38.0 & 57.5 & 1.5 & 2.3 & 90 & 0.13 & 23 & 2.44 & 2.8 & 23.6 & 0.30 & 37.4 & $3: 00$ & 6.0 \\
\hline L-13 & 26 & $\mathrm{~F}$ & 8.5 & 2.0 & 71.0 & 0.0 & 0.0 & 5.0 & 22.0 & 73.0 & 1.9 & 6.2 & 86 & 0.32 & 22 & 0.13 & 4.1 & 13.8 & 0.34 & 36.4 & $2: 00$ & 5.5 \\
\hline L-14 & 35 & $\mathrm{~F}$ & 5.8 & 2.0 & 46.0 & 1.0 & 2.0 & 9.0 & 40.0 & 51.0 & 2.3 & 3.0 & 86 & 2.47 & 23 & 1.79 & 2.6 & 13.0 & 0.32 & 36.9 & $2: 00$ & 3.5 \\
\hline MEAN & 37.6 & & 6.4 & 2.2 & 52.5 & 2.9 & 0.4 & 5.8 & 36.3 & 58.0 & 2.3 & 3.8 & 91 & 0.39 & 26 & 1.70 & 3.2 & 11.6 & 0.37 & 36.6 & & 6.2 \\
\hline
\end{tabular}

Group E (01-12): individuals with habit of going to sleep early (before midnight consistently); Group L (01-14): individuals with habit of staying up late (after 2 am. consistently). Sex M: male, Sex F: Female, WBC: white blood cell (leukocyte), Stab.: stab nuclear leukocyte, Seg.: segmented nuclear leukocyte, Eos.: Eosinophil, Baso.: Basophil, Mono.: monocyte, Lym.: lymphocyte, Gran.: granulocyte $(=$ Stab. + Seg. + Eos. + Baso. $)$, Number of Lym. $=\%$ Lym. $\times$ WBC, Number of Gran. $=\%$ Gran. $\times$ WBC, NA: Noradrenaline, FT3: free triiodothyronine.

Table 2 Summary of WBC, \%Lym., \%Gran., Number of Lym., Number of Gran., and Glucose in Group $E$ and $L$

\begin{tabular}{|c|c|c|c|c|c|c|}
\hline Subjects & $\begin{array}{c}\mathrm{WBC} \\
10^{3} / \mu \mathrm{L}\end{array}$ & $\begin{array}{c}\% \text { Lym. } \\
\%\end{array}$ & $\begin{array}{l}\% \text { Gran. } \\
\%\end{array}$ & $\begin{array}{c}\text { Number of Lym. } \\
10^{3} / \mu \mathrm{L}\end{array}$ & $\begin{array}{c}\text { Number of Gran. } \\
10^{3} / \mu \mathrm{L}\end{array}$ & $\begin{array}{l}\text { Glucose } \\
\mathrm{mg} / \mathrm{dL}\end{array}$ \\
\hline Group E & $6.0 \pm 0.31$ & $41.6 \pm 2.54 * *$ & $53.0 \pm 2.51^{* *}$ & $2.5 \pm 0.17 *$ & $3.2 \pm 0.27 *$ & $95 \pm 2.4^{*}$ \\
\hline Group L & $6.7 \pm 0.41$ & $31.7 \pm 2.03$ & $62.3 \pm 2.22$ & $2.1 \pm 0.13$ & $4.2 \pm 0.36$ & $88 \pm 2.7$ \\
\hline Total & $6.4 \pm 0.26$ & $36.3 \pm 1.84$ & $58.0 \pm 1.86$ & $2.3 \pm 0.11$ & $3.8 \pm 0.24$ & $91 \pm 1.9$ \\
\hline
\end{tabular}

Values are MEAN \pm SE. $* P<0.05 ; * * P<0.01$; significantly different from Group L. Other details are as in Table 1.

temperature, no statistically significant difference was found.

Difference in leukocyte subsets, blood glucose, and hormone levels due to length of time slept

Although Group E and Group L were separated by the time at which the subjects went to sleep, we also made comparison according to length of sleeping time. There was no statistically significant difference between length of sleeping time between Group E and Group L. When comparing the 15 sub- jects with length of time slept shorter than $6.2 \mathrm{~h}$, the average time of all 26 subjects, with the 11 subjects with length of time slept longer than $6.2 \mathrm{~h}$, no statistical significance was found for any test item (Tables 3, 4, and Fig. 2).

Difference in leukocyte subsets, blood glucose, and hormone levels due to difference in age and sexes of subjects

To compare leukocyte subsets, blood glucose, and hormone levels according to age, two groups were 

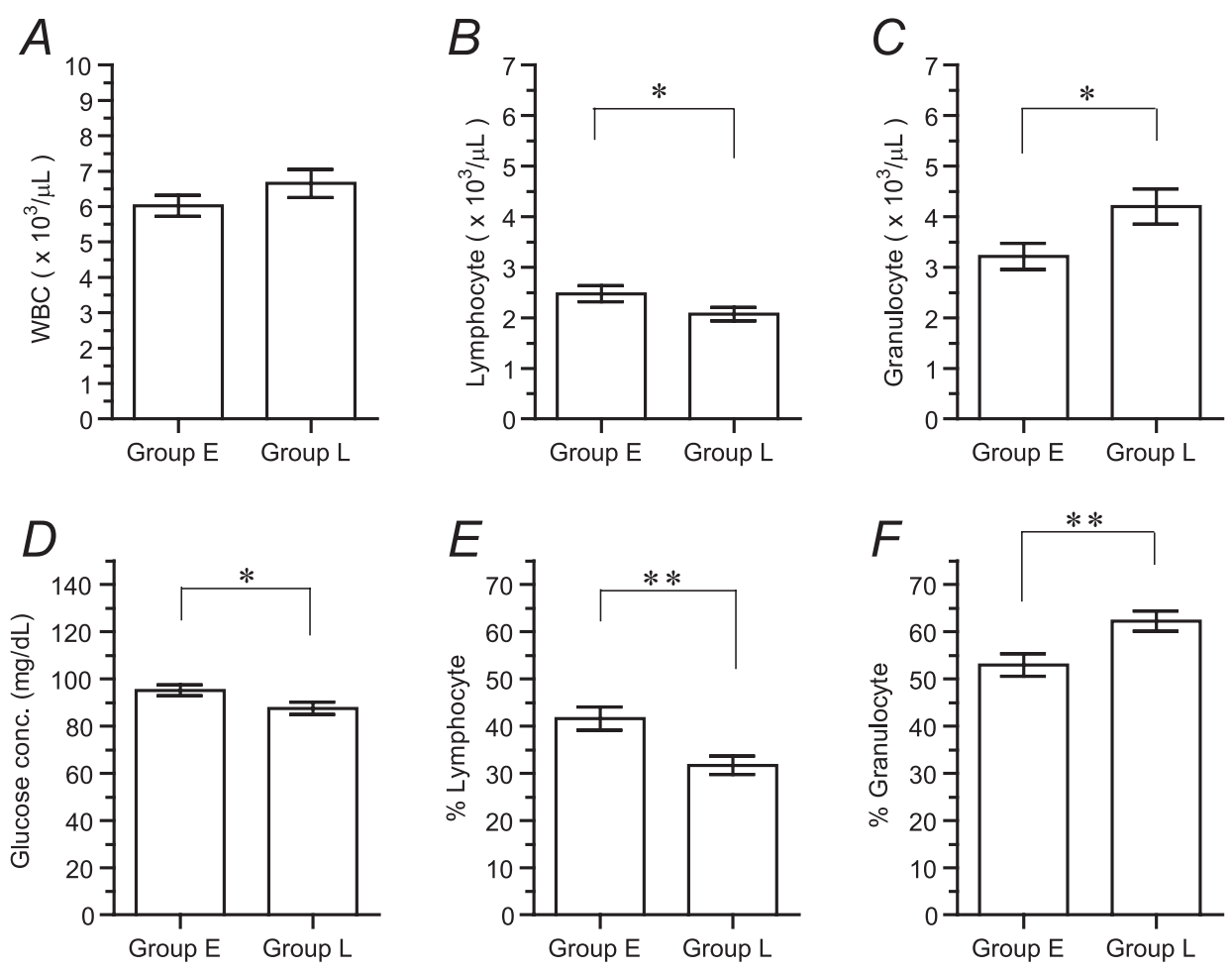

Fig. 1 Hematologic parameters, WBC (A); Lymphocyte (B); Granulocyte (C); Glucose concentration (D); \%Lymphocyte (E); \%Granulocyte $(\mathrm{F})$, in Group $\mathrm{E}$ and L. Each column and vertical bar represents the MEAN $\pm \mathrm{SE} .{ }^{*} P<0.05 ;{ }^{* *} P<0.01$

made according to age: for Group Y 14 subjects younger than the average age of all 26 subjects, 37.6 years old, and for Group O 12 subjects older than 37.6 years of age. For number of leukocytes, Group Y showed $6.5 \pm 0.41\left(\times 10^{3} / \mu \mathrm{L}\right)$ and Group O showed $6.2 \pm 0.33\left(\times 10^{3} / \mu \mathrm{L}\right)$; for ratio of lymphocytes Group Y showed $33.8 \pm 1.99 \%$ and Group O showed $39.1 \pm 3.23 \%$; for ratio of granulocytes Group Y was $59.9 \pm 2.20 \%$ and Group O was $55.8 \pm$ $3.19 \%$; for number of lymphocytes Group Y was 2.2 $\pm 0.15\left(\times 10^{3} / \mu \mathrm{L}\right)$ and Group $\mathrm{O}$ was $2.4 \pm 0.16$ $\left(\times 10^{3} / \mu \mathrm{L}\right)$; for number of granulocytes Group Y was $3.9 \pm 0.34\left(\times 10^{3} / \mu \mathrm{L}\right)$ and Group $\mathrm{O}$ was $3.5 \pm$ $0.37\left(\times 10^{3} / \mu \mathrm{L}\right)$; and for blood glucose level Group Y showed $89 \pm 3.0 \mathrm{mg} / \mathrm{dL}$ while Group $\mathrm{O}$ showed $93 \pm 2.5 \mathrm{mg} / \mathrm{dL}$. No statistically significant difference was found for any item. Also, for other hormones, just as in the case of comparing Group E and Group L, there was no significant difference found when comparing between age groups (between Group Y and Group O).

To compare according to different sexes, subjects were grouped into Group M consisting of 15 male subjects and Group F consisting of 11 female subjects and leukocyte subsets, blood glucose, and hormone levels were compared among the two groups.
For number of leukocytes, Group $\mathrm{M}$ was $6.7 \pm 0.33$ $\left(\times 10^{3} / \mu \mathrm{L}\right)$ and Group F was $5.9 \pm 0.42\left(\times 10^{3} / \mu \mathrm{L}\right)$; for ratio of lymphocytes, Group $\mathrm{M}$ was $36.2 \pm 2.39 \%$ and Group F was $36.3 \pm 3.16 \%$; for ratio of granulocytes Group $\mathrm{M}$ was $57.9 \pm 2.38 \%$ and Group $\mathrm{F}$ was $58.0 \pm 3.24 \%$; for number of lymphocytes Group $\mathrm{M}$ was $2.4 \pm 0.16\left(\times 10^{3} / \mu \mathrm{L}\right)$ and Group $\mathrm{F}$ was $2.1 \pm 0.13\left(\times 10^{3} / \mu \mathrm{L}\right)$; for number of granulocytes Group M was $3.9 \pm 0.29\left(\times 10^{3} / \mu \mathrm{L}\right)$ and Group $\mathrm{F}$ was $3.5 \pm 0.44\left(\times 10^{3} / \mu \mathrm{L}\right)$; and for blood glucose level Group M was $91 \pm 3.0 \mathrm{mg} / \mathrm{dL}$ while Group F was $91 \pm 2.2 \mathrm{mg} / \mathrm{dL}$. No statistically significant difference was found for any item. Also, for other hormones, just as in the case of comparing Group E and Group L, there was no significant difference found when comparing between sexes (between Group M and Group F).

\section{DISCUSSION}

In this research, we found that those with the habit of going to sleep early showed a higher ratio of lymphocytes while those with the habit of staying up late showed a higher ration of granulocytes. In order to demonstrate that the statistically significant difference among Group E and Group L as shown 
Table 3 Individual values of hematologic parameters, body temperatures, average times of going to sleep and average length of time slept in individuals with length of time slept shorter than $6.2 \mathrm{~h}$ and those with length of time slept longer than $6.2 \mathrm{~h}$

\begin{tabular}{|c|c|c|c|c|c|c|c|c|c|c|c|c|c|c|c|c|c|c|c|c|c|c|}
\hline \multirow[b]{2}{*}{ Group } & \multirow[b]{2}{*}{ Age } & \multirow[b]{2}{*}{ Sex } & \multirow[b]{2}{*}{$\begin{array}{c}\text { WBC } \\
10^{3} / \mu \mathrm{L}\end{array}$} & \multicolumn{7}{|c|}{$\%$ leukocyte subset count $(\%)$} & \multicolumn{2}{|c|}{ Number } & \multirow[b]{2}{*}{$\begin{array}{c}\text { Glucose } \\
\mathrm{mg} / \mathrm{dL}\end{array}$} & \multicolumn{6}{|c|}{ Hormone } & \multirow[b]{2}{*}{$\begin{array}{l}\text { Body } \\
\text { Temp. } \\
{ }^{\circ} \mathrm{C}\end{array}$} & \multirow[b]{2}{*}{$\begin{array}{c}\text { Time of } \\
\text { going to } \\
\text { sleep }\end{array}$} & \multirow[b]{2}{*}{$\begin{array}{l}\text { Length } \\
\text { of time } \\
\text { slept(h) }\end{array}$} \\
\hline & & & & Stab. & Seg. & Eos. & Baso. & Mono & Lym. & Gran. & $\begin{array}{c}\text { Lym. } \\
10^{3} / \mu \mathrm{L}\end{array}$ & $\begin{array}{c}\text { Gran. } \\
10^{3} / \mu \mathrm{L}\end{array}$ & & $\begin{array}{c}\mathrm{GH} \\
\mathrm{ng} / \mathrm{mL}\end{array}$ & $\begin{array}{l}\mathrm{ACTH} \\
\mathrm{pg} / \mathrm{mL}\end{array}$ & $\begin{array}{c}\text { TSH } \\
\mu \mathrm{IU} / \mathrm{mL}\end{array}$ & $\begin{array}{c}\text { FT3 } \\
\mathrm{pg} / \mathrm{mL}\end{array}$ & $\begin{array}{c}\text { Cortisol } \\
\mu \mathrm{g} / \mathrm{dL}\end{array}$ & $\begin{array}{c}\mathrm{NA} \\
\mathrm{ng} / \mathrm{mL}\end{array}$ & & & \\
\hline L-14 & 35 & $\mathrm{~F}$ & 5.8 & 2.0 & 46.0 & 1.0 & 2.0 & 9.0 & 40.0 & 51.0 & 2.3 & 3.0 & 86 & 2.47 & 23 & 1.79 & 2.6 & 13.0 & 0.32 & 36.9 & $2: 00$ & 3.5 \\
\hline L-02 & 19 & M & 7.0 & 3.5 & 51.5 & 3.5 & 0.0 & 4.5 & 37.0 & 58.5 & 2.6 & 4.1 & 92 & 0.15 & 15 & 4.36 & 3.5 & 8.3 & 0.36 & 36.8 & $5: 00$ & 4.5 \\
\hline E-08 & 45 & $\mathrm{~F}$ & 5.4 & 1.0 & 49.0 & 2.0 & 0.0 & 5.0 & 43.0 & 52.0 & 2.3 & 2.8 & 106 & 0.08 & 42 & 2.12 & 3.2 & 20.4 & 0.26 & 37.5 & $24: 00$ & 5.0 \\
\hline L-04 & 54 & M & 5.8 & 2.5 & 56.0 & 1.5 & 0.0 & 4.0 & 36.0 & 60.0 & 2.1 & 3.5 & 90 & 0.18 & 29 & 1.31 & 3.1 & 10.5 & 0.26 & 37.2 & $2: 00$ & 5.0 \\
\hline L-07 & 29 & M & 6.8 & 4.0 & 65.5 & 3.5 & 0.5 & 6.0 & 20.0 & 73.5 & 1.4 & 5.0 & 108 & 0.04 & 21 & 0.97 & 3.7 & 11.9 & 0.31 & 36.7 & $3: 00$ & 5.0 \\
\hline E-04 & 34 & M & 7.6 & 2.0 & 57.0 & 4.5 & 0.0 & 4.5 & 32.0 & 63.5 & 2.4 & 4.8 & 96 & 0.03 & 17 & 0.68 & 3.5 & 5.9 & 0.28 & 36.7 & $22: 30$ & 5.5 \\
\hline L-09 & 25 & $\mathrm{~F}$ & 6.1 & 4.0 & 51.0 & 4.0 & 0.0 & 5.0 & 36.0 & 59.0 & 2.2 & 3.6 & 83 & 0.16 & 24 & 1.66 & 3.2 & 10.8 & 0.17 & 35.4 & $5: 30$ & 5.5 \\
\hline L-13 & 26 & $\mathrm{~F}$ & 8.5 & 2.0 & 71.0 & 0.0 & 0.0 & 5.0 & 22.0 & 73.0 & 1.9 & 6.2 & 86 & 0.32 & 22 & 0.13 & 4.1 & 13.8 & 0.34 & 36.4 & $2: 00$ & 5.5 \\
\hline E-01 & 57 & F & 5.6 & 2.5 & 37.5 & 1.5 & 0.5 & 4.5 & 53.5 & 42.0 & 3.0 & 2.4 & 94 & 0.06 & 16 & 3.01 & 3.2 & 6.7 & 0.83 & 36.3 & $24: 00$ & 6.0 \\
\hline E-02 & 21 & M & 6.7 & 4.0 & 36.0 & 6.0 & 0.0 & 8.0 & 46.0 & 46.0 & 3.1 & 3.1 & 104 & 0.14 & 47 & 1.57 & 3.1 & 18.6 & 0.29 & 36.7 & $24: 00$ & 6.0 \\
\hline E-06 & 53 & $\mathrm{~F}$ & 4.4 & 1.0 & 43.5 & 1.5 & 1.0 & 5.5 & & 47.0 & 2.1 & 2.1 & 97 & 0.09 & 18 & 1.08 & 3.1 & 9.9 & 0.42 & 36.7 & $24: 00$ & 6.0 \\
\hline E-09 & 59 & M & 7.6 & 1.0 & 53.0 & 2.0 & 0.0 & 3.0 & 41.0 & 56.0 & 3.1 & 4.3 & 97 & 0.05 & 77 & 1.62 & 3.1 & 11.7 & 0.41 & 36.0 & $23: 30$ & 6.0 \\
\hline E-12 & 38 & M & 6.3 & 1.0 & 56.0 & 3.0 & 1.0 & 9.0 & 30.0 & 61.0 & 1.9 & 3.8 & 86 & 0.26 & 13 & 2.11 & 3.3 & 9.8 & 0.39 & 36.3 & $23: 00$ & 6.0 \\
\hline L-01 & 51 & M & 7.9 & 4.5 & 62.5 & 1.5 & 0.0 & 5.0 & 26.5 & 68.5 & 2.1 & 5.4 & 79 & 0.04 & 24 & 1.46 & 3.3 & 8.5 & 0.43 & 36.2 & $3: 00$ & 6.0 \\
\hline $\mathrm{L}-12$ & 28 & $\mathrm{~F}$ & 4.0 & 3.0 & 52.5 & 1.0 & 1.0 & 4.5 & 38.0 & 57.5 & 1.5 & 2.3 & 90 & 0.13 & 23 & 2.44 & 2.8 & 23.6 & 0.30 & 37.4 & $3: 00$ & 6.0 \\
\hline E-05 & 30 & $\mathrm{~F}$ & 4.9 & 3.0 & 56.0 & 0.5 & 0.5 & 6.0 & 34.0 & 60.0 & 1.7 & 2.9 & 84 & 0.41 & 13 & 1.49 & 2.7 & 8.2 & 0.33 & 36.9 & $24: 00$ & 6.5 \\
\hline E-03 & 40 & M & 5.3 & 1.0 & 45.5 & 1.0 & 0.0 & 8.0 & 44.5 & 47.5 & 2.4 & 2.5 & 91 & 0.34 & 52 & 1.86 & 3.5 & 13.0 & 0.36 & 35.7 & $24: 00$ & 7.0 \\
\hline L-05 & 51 & $\mathrm{~F}$ & 7.7 & 3.5 & 69.0 & 2.5 & 0.0 & 5.0 & 20.0 & 75.0 & 1.5 & 5.8 & 88 & 2.04 & 12 & 0.80 & 2.7 & 8.2 & 0.51 & 35.7 & $2: 00$ & 7.0 \\
\hline L-06 & 20 & M & 6.3 & 1.0 & 50.0 & 2.0 & 0.0 & 6.5 & 40.5 & 53.0 & 2.6 & 3.3 & 100 & 0.18 & 19 & 1.13 & 3.5 & 9.6 & 0.28 & 36.9 & $3: 00$ & 7.0 \\
\hline L-08 & 24 & M & 5.9 & 1.5 & 46.5 & 6.0 & 1.0 & 9.5 & 35.5 & 55.0 & 2.1 & 3.2 & 77 & 0.36 & 41 & 1.65 & 3.1 & 15.7 & 0.73 & 36.8 & $4: 00$ & 7.0 \\
\hline L-10 & 26 & M & 5.5 & 2.5 & 65.0 & 0.0 & 0.5 & 5.5 & 26.5 & 68.0 & 1.5 & 3.7 & 68 & 0.24 & 15 & 1.54 & 3.5 & 13.2 & 0.39 & 37.0 & $2: 00$ & 7.0 \\
\hline L-11 & 31 & $\mathrm{~F}$ & 5.9 & 1.0 & 54.0 & 1.0 & 0.0 & 9.0 & 35.0 & 56.0 & 2.1 & 3.3 & 94 & 0.15 & 18 & 2.86 & 2.8 & 14.4 & 0.27 & 36.4 & $3: 00$ & 7.0 \\
\hline E-07 & 45 & $\mathrm{~F}$ & 6.8 & 1.0 & 59.0 & 5.0 & 1.0 & 4.0 & 30.0 & 66.0 & 2.0 & 4.5 & 97 & 0.88 & 25 & 1.83 & 3.0 & 10.4 & 0.28 & 36.7 & $24: 00$ & 7.5 \\
\hline L-03 & 31 & M & 10.0 & 3.0 & 54.5 & 6.5 & 0.0 & 5.5 & 30.5 & 64.0 & 3.1 & 6.4 & 84 & 0.04 & 23 & 0.62 & 3.4 & 6.4 & 0.36 & 37.1 & $2: 30$ & 7.5 \\
\hline E-10 & 56 & M & 5.4 & 0.5 & 40.0 & 10.5 & 1.5 & 4.0 & 43.5 & 52.5 & 2.3 & 2.8 & 84 & 1.21 & 17 & 3.03 & 2.6 & 4.2 & 0.37 & 36.0 & $23: 00$ & 8.0 \\
\hline E-11 & 49 & M & 6.3 & 1.0 & 38.0 & 3.0 & 0.0 & 4.0 & 54.0 & 42.0 & 3.4 & 2.6 & 106 & 0.09 & 21 & 1.04 & 3.4 & 14.6 & 0.35 & 36.4 & $24: 00$ & 8.0 \\
\hline MEAN & 37.6 & & 6.4 & 2.2 & 52.5 & 2.9 & 0.4 & 5.8 & 36.3 & 58.0 & 2.3 & 3.8 & 91 & 0.39 & 26 & 1.70 & 3.2 & 11.6 & 0.37 & 36.6 & & 6.2 \\
\hline
\end{tabular}

Details are as in Table 1 .

Table 4 Summary of WBC, \%Lym., \%Gran., Number of Lym., Number of Gran., and Glucose in individuals with length of time slept shorter than $6.2 \mathrm{~h}$ and those with length of time slept longer than $6.2 \mathrm{~h}$

\begin{tabular}{ccccccc}
\hline & WBC & $\begin{array}{c}\text { \% Lym. } \\
\%\end{array}$ & $\begin{array}{c}\text { \% Gran. } \\
\%\end{array}$ & $\begin{array}{c}\text { Number of Lym. } \\
10^{3} / \mu \mathrm{L}\end{array}$ & $\begin{array}{c}\text { Number of Gran. } \\
10^{3} / \mu \mathrm{L}\end{array}$ & $\begin{array}{c}\text { Glucose } \\
\mathrm{mg} / \mathrm{dL}\end{array}$ \\
\hline Shorter than $6.2 \mathrm{~h}$ & $6.4 \pm 0.34$ & $36.6 \pm 2.50$ & $57.9 \pm 2.51$ & $2.3 \pm 0.14$ & $3.8 \pm 0.33$ & $93 \pm 2.3$ \\
Longer than $6.2 \mathrm{~h}$ & $6.4 \pm 0.45$ & $35.8 \pm 2.98$ & $58.1 \pm 3.05$ & $2.2 \pm 0.19$ & $3.7 \pm 0.41$ & $88 \pm 3.4$ \\
\hline Total & $6.4 \pm 0.26$ & $36.3 \pm 1.84$ & $58.0 \pm 1.86$ & $2.3 \pm 0.11$ & $3.8 \pm 0.24$ & $91 \pm 1.9$ \\
\hline
\end{tabular}

Details are as in Tables 1 and 2.

in Table 2 was not affected by differences in age or sex, we also performed statistical analysis across groups divided by age and by sex. The results of this analysis showed that there was no statistically significant difference when grouping by age or sex. These analyses clearly show that the difference in leukocyte subsets (lymphocytes and granulocytes) was due to the difference in the time at which the subjects went to sleep. When discussing the effects of sleep on the body, many tend to speak of the length of time slept, but in this research we did not find a statistically significant effect on leukocyte subsets due to differences in length of time slept.
However, a large change in leukocyte subsets was shown to result from the difference in the time that the subjects went to sleep. In other words, the time at which a person goes to sleep, one important factor in determining one's overall body rhythm, also has a large effect on the composition of one's leukocyte subset-a greater amount of granulocytes, or a greater amount of lymphocytes.

It has been reported in the past that granulocytes present adrenaline receptors on the outer membranes, and that adrenaline injections can cause an excess of granulocytes. And it may be the case that the sympathetic nervous system acts to control the 

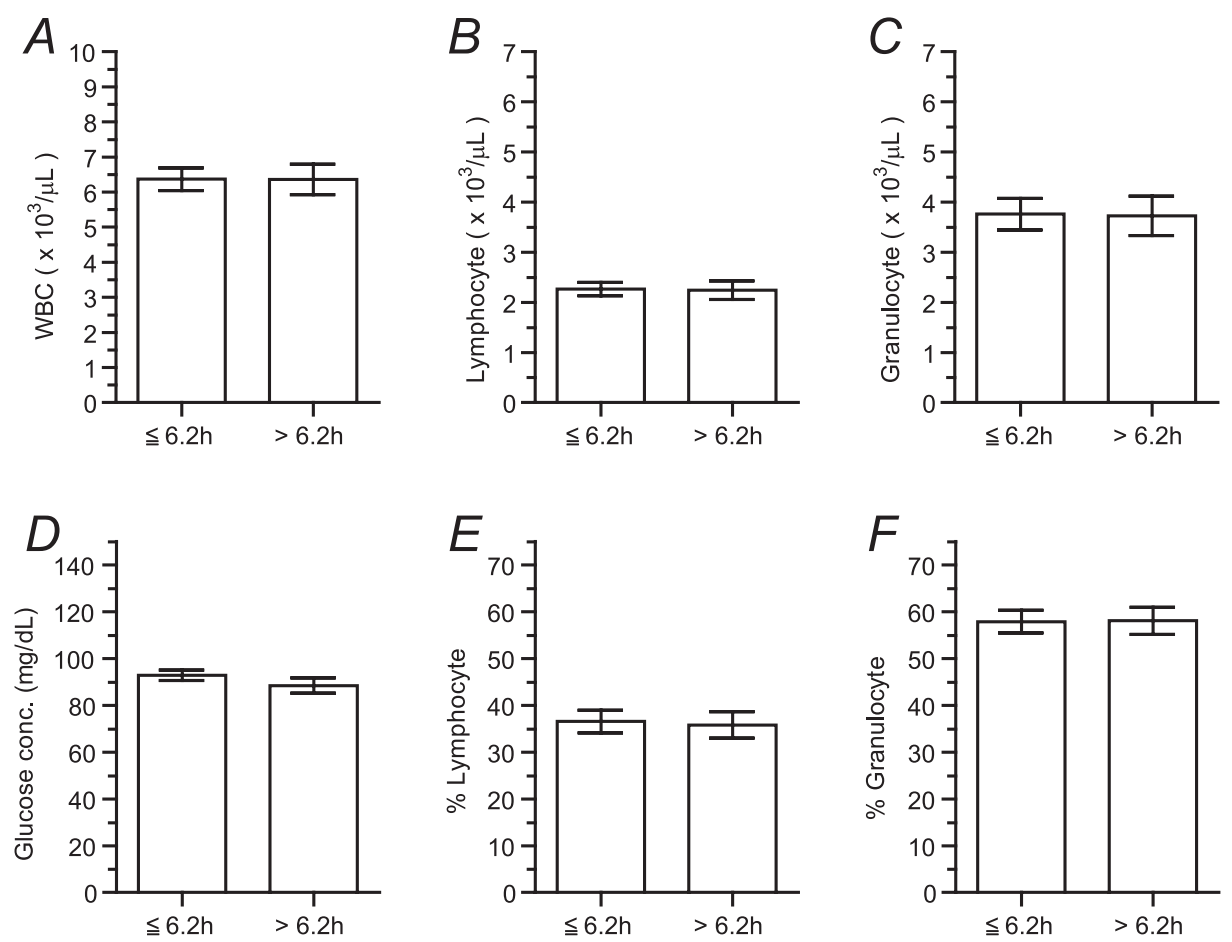

Fig. 2 Hematologic parameters, WBC (A); Lymphocyte (B); Granulocyte (C); Glucose concentration (D); \%Lymphocyte (E); $\%$ Granulocyte $(\mathrm{F})$, in individuals with length of time slept shorter than $6.2 \mathrm{~h}$ and those with length of time slept longer than $6.2 \mathrm{~h}$. Each column and vertical bar represents the MEAN $\pm \mathrm{SE}$.

number of granulocytes $(3,4)$. These reports thus show agreement with our result that those with the habit of staying up until late at night, causing stimulation of the sympathetic nervous system until late at night, showed an excess of granulocytes. However, another result of our research, that the levels of noradrenaline in the blood of those with the habit of staying up late, and thus with stimulation of the sympathetic nervous system until late at night, were not significantly different than the levels of noradrenaline in the blood of those with the habit of going to sleep early, shows that the excess of granulocytes found in those staying up late is not from noradrenaline in the blood, but rather from a different cause. Also, in this research no statistically significant difference was found for the other various hormones in the blood as tested, so the excess of granulocytes in those with the habit of staying up late is not from these hormones either.

In this research, the blood glucose level for Group L was significantly lower than the blood glucose level for Group E. A previous report has noted the relationship between diabetes and length of time slept (1). However, this report focuses on length of time slept, not the time at which subjects first went to sleep as in our study. To offer one hypothesis from our research, perhaps it is the case that among those with the habit of staying up late, this results in impairment of the autonomic nervous system and subsequently a decrease in blood sugar during the day, leading to more frequent feelings of hunger, and the resulting irregular consumption of food then invites the onset of diabetes.

In addition, from our research it can be seen that the practice of sleeping for a longer time in order to make up for going to sleep at a later hour does not offset the trend toward increased number of granulocytes. In other words, the factor driving the difference in leukocyte subsets is not the length of time slept but rather the adaptation of one's day/night body rhythms.

\section{REFERENCES}

1. Gottlieb DJ, Punjabi NM, Newman AB, Resnick HE, Redline S, Baldwin CM and Nieto FJ (2005) Association of sleep time with diabetes mellitus and impaired glucose tolerance. Arch Intern Med 165, 863-868.

2. Panosian JO and Marinetti GV (1983) $\alpha_{2}$-Adrenergic receptors in human polymorphonuclear leukocyte membranes. Biochem Pharmacol 32, 2243-2247.

3. Suzuki S, Toyabe S, Moroda T, Tada T, Tsukahara A, Iiai T, Minagawa M, Maruyama S, Hatakeyama K, Endoh K and Abo T (1997) Circadian rhythm of leucocytes and lympho- 
cyte subsets and its possible correlation with the function of the autonomic nervous system. Clin Exp Immunol 110, 500508.

4. Tsukahara A, Tada T, Suzuki S, Iiai T, Moroda T, Maruyama S,
Minagawa M, Musha N, Shimizu T, Hatakeyama K and Abo

T (1997) Adrenergic stimulation simultaneously induces the expansion of granulocytes and extrathymic $\mathrm{T}$ cells in mice. Biomed Res 18, 237-246. 
